\section{New OA management guidelines}

The American College of Rheumatology (ACR) and the Arthritis Foundation have provided new evidence-based guidelines for the management of osteoarthritis (OA) of the hands, hip and knees, updating previous ACR recommendations from 2012. The new guidelines include strong recommendations for exercise, self-efficacy and self-management programmes, as well as oral NSAIDs.

Unlike the previous 2012 recommendations, which relied primarily

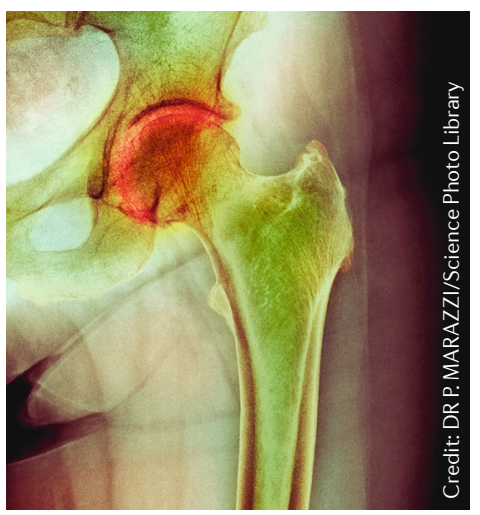

on externally performed systematic reviews and meta-analyses, the development of the new guidelines included a comprehensive systematic literature review. "We had the same team working on the entire literature review producing one methodologically consistent meta-analysis of all of the relevant literature," explains corresponding author Sharon Kolasinski.

The quality of the evidence was rated using Grading of Recommendations Assessment, Development and Evaluation methodology. "One innovation of our process compared to the last guideline was the convening of a patient panel who reviewed the evidence report and commented on all of the Kolasinski. "The patients involved had a very broad personal experience with many of the interventions and were invaluable in contributing their perspective, particularly on controversial interventions." interventions considered," notes

The voting panel, consisting of rheumatologists, an internist, physical and occupational therapists, and two representatives from the patient panel, made conditional recommendations for and against various therapies for OA, including educational, behavioural, psychosocial, physical, mindbody and pharmacological therapies.

"The biggest impact to practice might come from the conditional recommendation against hyaluronic acid injections," says Kolasinski. Hyaluronic acid injections were previously conditionally recommended for use in knee OA. "The best evidence suggests that hyaluronic acid injections are equivalent to saline injections in efficacy," she explains.

Overall, the new guidelines emphasize the importance of shared decision making between clinicians and patients and highlight gaps in our knowledge that need addressing in future studies.

Jessica McHugh

ORIGINAL ARTICLE Kolasinski, S. L. et al. 2019 American College of Rheumatology/Arthritis Foundation Guideline for the Management of Osteoarthritis of the Hand, Hip, and Knee. Arthritis Rheum. https://doi.org/10.1002/art.41142 (2019)

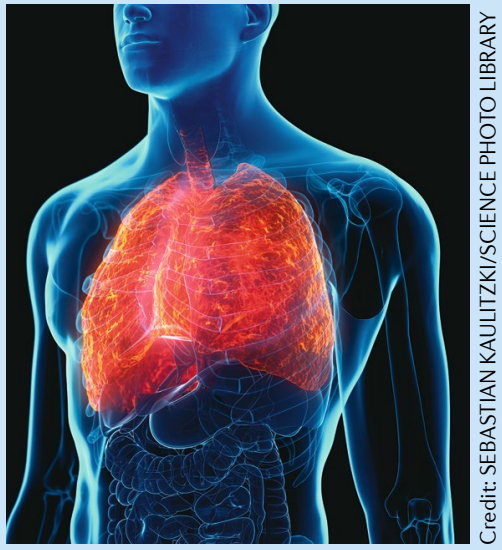

"There are still many unmet needs, but once we are getting more good quality studies, work on guidelines for the identification and management of SSc-associated ILD should be initiated," all patients with SSc should be screened using highresolution $\mathrm{CT}$ when to initiate or escalate these in SSc-associated ILD," says corresponding author Anna-Maria Hoffmann-Vold. The authors recommend that highresolution CT should be the primary tool for the diagnosis of ILD, supported by pulmonary function tests and clinical assessments of respiratory symptoms. and that all patients with $\mathrm{SSc}$ should With regard to treatment, the authors recommend that all patients with severe lung disease receive pharmacological treatment and suggest that mycophenolate mofetil or cyclophosphamide can be effective, as can the novel therapeutic nintedanib. By contrast, they suggest that those with mild disease should be monitored closely, but not necessarily receive drug therapy. The consensus statement also covers monitoring of disease progression and alternative treatment options such as haematopoietic stem cell or lung transplantation. these emerging treatment options and

\section{guidelines of shared}

SYSTEMIC SCLEROSIS treatment initiation and options, disease progression and treatment escalation). \section{concludes Hoffmann-Vold. \\ Joanna Clarke \\ concludes Hoffmann-Vold.}

ORIGINAL ARTICLE Hoffmann-Vold, A.-M. et al. The identification and management of interstitial lung disease in systemic sclerosis: evidence-based European consensus statements. Lancet Rheumatol. https://doi.org/10.1016/S2665-9913(19)30144-4 (2020)

RELATED ARTICLE Denton, C. P. et al. Major lung complications of systemic sclerosis. Nat. Rev. Rheumatol. 14, 511-527 (2018) 